\title{
On the Stability and Design of Distributed Manipulation Control Systems
}

\author{
T. D. Murphey, J. W. Burdick \\ Engineering and Applied Science, California Institute of Technology \\ Mail Code 104-44, Pasadena, CA 91125 USA \\ \{murphey,jwb\}@ robotics.caltech.edu
}

\begin{abstract}
This paper analyzes the stability of distributed manipulation control schemes. A commonly proposed method for designing a distributed actuator array control scheme assumes that the system's control action can be approximated by a continuous vector force field. The continuous control vector field idealization must then be adapted to the physical actuator array. However, we show in this paper that when one takes into account the discreteness of actuator arrays and realistic models of the actuator/object contact mechanics, the controls designed by the continuous approximation approach can be unstable. For this analysis we introduce and use a "power dissipation" method that captures the contact mechanics in a general but tractable way. We show that the quasi-static contact equations have the form of a switched hybrid system. We introduce a discontinuous feedback law can produce stability which is robust with respect to variations in contact state.
\end{abstract}

\section{Introduction and Previous Work}

A distributed manipulation system consists of an (roughly planar) array of actuators that can reposition an object by the movements of its array elements (see Fig. 1). In the future, arrays of this type should be usefu.l for industrial assembly operations where small parts must be robustly transported and precisely positioned. This paper considers the design of manipulation control strategies for such distributed systems. We focus on autonomous controllers that stabilize an object to a precise configuration equilibrium on the array.

Methods to design distributed nanipulation control systems have been proposed in several works, including Refs. $[1,2]$. A common approach is based on the notion of programmable vector fields [3]. In this methodology, one makes the possibly unrealistic assumption that the array's control capability can be idealized as a continuous distribution of forces across the array surface. In this abstraction, the manipulated object moves under the influence of these forces. The control design problem reduces to the selection of a continuous force field distribution that will locally transport the object to a prescribed position, and then stabilize it at that configuration. To implement the control strategy on the real array, one must adipt the continuous vector field control to the real (and discrete) actuator array. For a

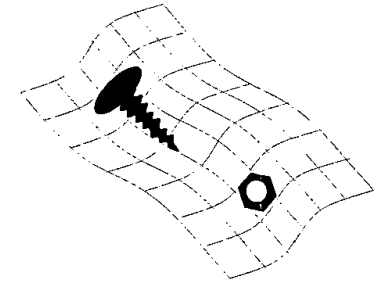

Figure 1: Parts on a distributed manipulator good description of this approach, see Bohringer et al [3].

This approach is experimentally known to work in MEMS-fabricated actuator arrays, where the array elements are "small" and "close" together [3]. However, in cases where the actuators are far apart (i.e., the continuous actuation approximation is poor) or the coefficient of friction $\mu$ is very high, the continuous approximation is known not to work as well (see Luntz et al [4]). In these cases, the continuous approximation does not adequately incorporate the physics of the actual array and the object/array interface.

This paper has three main contributions. First we show that when one takes into account the discrete nature of real actuator arrays and a fairly general model of the actuator-toobject contact mechanics, the control systems designed by the continuous approximation method can often be unstable when deployed on the actual array. This is not unexpected, as the programmable vector field approach is based on the restrictive assumption that the continuous vector field abstraction is a good approximation to the array's açtual physical characteristics. This instability result has been previously shown for specific array geometries [4]. This paper generalizes work done by Luntz et al [4] in that where they considered a specific model with smooth dynamics, we have instability results which take into account contact mechanics. Here we show this instability for a quite general class of actuator arrays.

Secondly, we discuss a power dissipation methodology for modeling the array/object contact. This method, which is adapted from the work of Alexander and Maddocks [5] in the area of wheeled vehicles, is based on the principle that an object will move in the direction that minimizes the power dissipation associated with moving. This method applies to fairly general types of array/object contact, and it 
results in tractable models. We formalized this approach in Ref. [6], showing that actuator/object contact models take the form of a switched hybrid control system. This observation allows tools from non-smooth analysis and the study of differential inclusions to be applied to this problem. The suitability of this approach is discussed in Section 2. In it, we assume that rather than having a force field, $F(x, y)$ defined everywhere in the plane, we have instead a velocity field $\Psi(x, y)$ defined everywhere. Intuitively, this corresponds to integrating the distinct actuator forces up to velocities and controlling those directly. Finally, we introduce a control scheme to stabilize an object on a distributed array, and use our power dissipation model to prove the scheme's stability in the quasi-static case.

Section 3 shows by way of an example how controllers designed by the programmable vector field paradigm can be unstable. As in [4], our example models the Modular Distributed Manipulator System (MDMS). We show that the object's rotational dynamics are unstable, and that feedback is capable of stabilizing the object. Section 3 also introduces a stabilizing control law for this system. Section 4 provides a theorem that generalizes the example of Section 3 to a larger set of array geometries. Section 5 presents illustrative simulation results.

\section{The Power Dissipation Model}

We seek models for distributed actuation systems that faithfully capture the system's essential physics, and that are tractable and amenable to control and motion planning analysis. In pursuit of this goal, we use a power dissipation approach to model the discrete actuator/object system. This method typically produces unique models that are relatively easy to obtain. Since the method is a quasi-static modeling method, it produces first-order governing equations, instead of second order equations that are associated with Lagrange's equations. The primary disadvantage is that the method only applies to quasi-static systems. Below we argue that this assumption is quite good for our problems of interest, and for distributed manipulation arrays in general.

We assume throughout that the object's contact with the manipulating surface is governed by the Coulomb friction law at each point of contact. We treat the object and the array element contact as a rigid body contact system (though approximate compliance effects can be easily incorporated into this paradigm). Let us assume without loss of generality that the object is in contact with several array elements. Let us further assume that the contact with each array element is a point contact, or set of point contacts (complex contacts such as line contacts are modeled as a set of point contacts in this approach). With sufficiently many contacts between the object and the manipulating surface, it will often be true that one or more contacts must slip during object motion, thereby dissipating energy. I.e., no motion exists where all of the contacts can be simultaneously slipless. The power dissipation method assumes that the object's motion at any given instant is the one that instanta- neously minimizes power dissipation due to contact slippage. This method is adapted from the work of Alexander and Maddocks [5] on wheeled vehicles.

Let $q$ denote the configuration of the array/object system, consisting of the object's planar location, and the variables that describe the state of each actuator array element. Let us assume that the motion of the actuator array's variables are known. It is not hard to show that the relative motion of each contact between the object and an actuator array element can be modeled in the form $\omega(q) \dot{q}$. If $\omega(q) \dot{q}=0$, then the contact is not slipping (i.e., it is nonholonomic), while if $\omega(q) \dot{q} \neq 0$, then $\omega(q) \dot{q}$ describes the slipping velocity. The power dissipation function measures the object's total energy dissipation due to contact slippage.

Definition 1 The Dissipation or Friction Functional for an $n$-contact state is defined to be

$$
\mathcal{D}=\sum_{i=1}^{n} \alpha_{i}|\omega(q) \dot{q}|
$$

where $\alpha_{i}=\mu_{i} N_{i}$, with $\mu_{i}$ and $N_{i}$ being the Coulomb friction coefficient and normal force at the $i^{\text {th }}$ contact, which are assumed known.

Ideally, one would develop a model using a complete constrained Lagrangian mechanics approach. However, the Lagrangian approach leads to a great deal more complexity than the PDM approach, and with little insight if the problem is roughly quasi-static. Additionally, the PDM approach will lead to the same contact states that the Lagrangian method will lead to in the case of slow speeds (or sufficiently small input forces). We now argue that the quasi-static assumption is quite appropriate for our analysis, and will generally hold for most distributed manipulation systems of interest. First, our analysis is generally concerned with the stability of the object's motion near the object's equilibrium point. The quasi-static assumption is an excellent approximation for these conditions.

More generally, the assumptions underlying the PDM approach are reasonable for most practical distributed manipulation systems. To see this, consider a block moving on top of two contacts, A and B (we can extend this discussion to more contacts). Then an analysis based on Lagrangian mechanics would suggest that there are up to four possible contact states, corresponding to a) A slipping, b) B slipping, c) neither slipping, $d$ ) both slipping. On the other hand, the PDM analysis finds that there are only two different contact states corresponding to either A or B slipping. Possibilities c) and d) both imply that the inertial terms dominate the system's dynamics, thereby violating the quasi-static assumption. It is unlikely the dynamic states corresponding to d) will be practically realized in a distributed manipulation system, as they correspond to conditions where the object is skidding across the manipulation surface-i.e., it is not under control. Similarly, the conditions corresponding to state c) are unlikely to be found in a distributed manipulation system, as this implies that both contacts must be driven at exactly compatible speeds, or the normal forces must be 
so high that they affect the input speeds. In the case of c) where the speeds are exactly the same speed, the dimension of the subspace spanned by the constraints drops in any case, implying that the constraint is essentially duplicated. Therefore the power dissipation model will give results satisfying this constraint even if it is practically unlikely. This leaves the second two states, which are the same as what we found in the power dissipation model. This is an indication of how the quasi-static assumption, which will be valid near the equilibrium of a distributed system (and more generally in most practical distributed manipulation systems), helps to simplify our problem. To characterize the properties of the models obtained using the PDM, the next section reviews some of the previous formal results that the authors have derived for this scheme.

\subsection{The Power Dissipation Model Leads to Switched Hybrid Systems.}

Here we briefly summarize some of the PDM's formal characteristics, which were first developed in the context of wheeled vehicles. In particular, 'we show that the power dissipation approach generically leads to switched hybrid systems. For the omitted proofs and a greater discussion of these results, see Ref. [6]. We first consider the extent to which the function $\mathcal{D}$ having a unique minimum (and therefore a uniquely defined set of equations governing the object's motion) is generic.

Theorem 1 Assume $\mathcal{D}$ is of the form in Definition 1 and that the $\mu$ is measurable in $x$ and $t$. Then the dissipation functional $\mathcal{D}$ has a unique minimum almost always (i.e. except on a set of measure 0 )

That is, the PDM will almost always lead to a unique set of governing equations. Even in the non-generic case where the minimum of $\mathcal{D}$ is not unique, we have the following.

Theorem 2 If $\dot{q}_{1}$ and $\dot{q}_{2}$ both minimize the dissipation functional found in Definition 1, then so does $\operatorname{co}\left\{\dot{q}_{1}, \dot{q}_{2}\right\}$.

This result formalizes the intuition that if the power dissipated is equal for two velocities $\dot{q}_{i}$, then all possible trajectories whose velocity lies in the convex hull of the $\dot{q}_{i}$ will satisfy the minimum also. I.e., in the non-generic case when $\mathcal{D}$ does not have a unique minimum, we can still bound the object's motion. Our next definition describes the kind of system one obtains by using the PIDM approach.

Definition 2 A system is a switched driftless affine system (SDA) if it can be expressed in the form

$$
\dot{x}=f_{\sigma_{1}}(x) u_{1}+f_{\sigma_{2}}(x) u_{2}+\cdots+f_{\sigma_{n}}(x) u_{n}
$$

where for any $x$ and $t, f_{\sigma_{i}}(x) \in\left\{g_{\alpha_{i}}(x) \mid \alpha_{i} \in I_{i}\right\}$, with $I_{i}$ an index set and $f_{i}$ measurable ir: $(x, t)$ and $g_{i}$ analytic in $(x, t)$ for all $i$.

An SDA is a driftless affine nonlinear control system where each control vector fields may "switch" back and forth between different elements of a finite set. In our case, this switching corresponds to the switching between different contact states between the object and the array surface elements (i.e., different sets of slipping contacts). In [6] it was shown that the PDM generically leads to switched driftless affine systems as in Definition 2 and that this representation is generically unique.

\section{Modeling the Equilibrium Point of a Pro- grammable Vector Field}

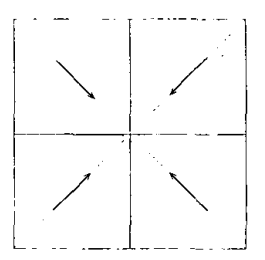

Figure 2: Four node array centered at the origin

Here we apply the PDM technique to a specific example in order to show that if we use the model obtained by the PDM, we can get rotational instability in part placement when the passive programmed vector field approach, as described in [7], is applied to this system. The next section generalizes this result to a broader class of actuator arrays.

The difference between the stability prediction of the continuous approximation and the more exact model rests largely on the fact that the continuous approximation does not take any contact mechanics into account, nor does it account for the fact that most real arrays consist of a finite number of discrete actuators. Of course, the PDM can only be well justified for quasi-static systems where the objects move slowly enough that the contact reaction forces dominate the moments of inertia. This will be true in our case, where we study the local behavior of object motion around an equilibrium point. We consider a relatively simple example of just four actuator array elements (see Figure 2). The actuating elements consist of a wheel whose rim points towards the origin and whose speed is controllable. The wheel is assumed to make point contact with the body at all times.

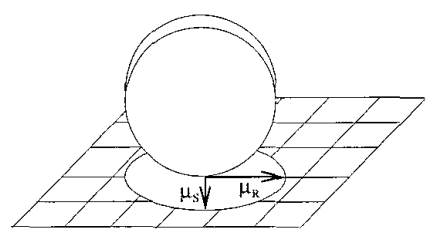

Figure 3: A wheel with anisotropic friction

Generally, there is no reason to believe that friction at each contact point will be uniform in all directions of the contact plane. Rather, we allow a smooth distribution of coefficient of friction, like that seen in Figure 3 (see Ref. [8] for a discussion of such friction models). While some materials do have friction of this type, such anisotropic friction models are more generally useful as a means to approximately model compliance effects and wheel tread effects. For instance, if the wheel shown above was as thin as a saw blade, then we would expect $\mu_{R}$ (the friction coefficient along the "rim" direction) to be less than $\mu_{S}$ (the friction coefficient along the "side" direction), and in doing so 
we would be modeling the non-ideal point contact in terms of a variable coefficient of friction. However, the treads on a tank ensure that $\mu_{R}$ is greater than $\mu_{S}$. Note that the minimum of the dissipation function will only be non-unique when the ellipse reduces to a circle (i.e. $\mu_{S}=\mu_{R}$ ). Moreover, the same indeterminacy shows up in the analogous Lagrangian analysis.

\subsection{Equations of Motion}

For the sake of brevity, this section sketches, at an intuitive level, the application of the power dissipation method to this example. Let $\Psi$ be a velocity field taking configurations to actuator input speeds, i.e. $\Psi: Q \rightarrow \mathbb{R}$. We assume that the equilibrium point of $\Psi(x, y)$ lies at the intersection point of the lines underlying the wheel rims.

With four wheel actuators, there is a potential total of 8 kinematic constraints on the objects motion. However, since the object moves in the plane, at most three of these constraints can be generically satisfied at any instant-the other constraints must be violated via contact slipping (except in special configurations). This gives us $\left(\begin{array}{l}8 \\ 3\end{array}\right)=56$ possible kinematics. Of course, many of these are incompatible with each other. For instance, satisfying three no sideways slip constraints is not typically meaningful since that would entail the object's contact state being dominated by three different actuators. Of these 56 possible kinematics, only 8 ever satisfy the minimum of the power dissipation function on a set of full measure, therefore leaving us with a total of 8 possible contact states. The constraints associated with each actuator are $\Omega(q) \dot{q}=0$ where

$$
\Omega_{i}(q)=\left[\begin{array}{c}
A d_{g_{i}^{-1}}^{T}\left(\begin{array}{l}
1 \\
0 \\
0 \\
0 \\
A d_{g_{i}^{-1}}^{T}\left(\begin{array}{l}
1 \\
0
\end{array}\right)
\end{array}\right) \Psi(x, y) \\
0
\end{array}\right]
$$

and

$$
g_{i}=\left[\begin{array}{cc}
R\left(\theta_{i}\right) & \left(\begin{array}{c}
x_{i} \\
y_{i} \\
1
\end{array}\right) \\
0 & 1
\end{array}\right] \in S E(2)
$$

is the homogeneous representation of the $i$ th actuator node location and orientation relative to a fixed reference frame, and $A d(\cdot)$ is the adjoint transformation which transforms velocities from one coordinate frame to another.

Now to apply the PDM to the above set up, first note that the minimum only occurs when three of the constraints are satisfied, and that moreover, the constraints satisfied are precisely those which would otherwise dissipate the most energy if they were violated. The contact states that dissipate the most energy are those associated with the potential constraints having the largest three normal forces $\alpha_{i}=N_{i} \mu_{i}$. Based on these observations, if the center of mass determines the normal forces (based on assumptions about surface uniformity, etc.), and if $\mu(x, y)$ is uniform, then the object's motion satisfies whichever constraints are closest to its center of mass. That is, the particular quadrant in which the center of mass lies determines the first two actively satisfied constraints. The third actively satisfied constraint is determined by the friction model, and whichever side of the diagonal $y=x$ the center of mass lies. When $\mu_{S}>\mu_{R}$, the system equations are found by solving for the anhilator of the constraint $\Omega(q)$. Assuming unit magnitude input in each actuator, solving each set of constraints, and doing some algebraic simplification yields the following governing equations:

$$
\left[\begin{array}{l}
\dot{x} \\
\dot{y} \\
\dot{\theta}
\end{array}\right]=\left[\begin{array}{c}
\left.-\frac{\sqrt{2}}{2} \operatorname{sgn}(x)\left(\operatorname{sgn}\left(y^{2}-x^{2}\right)+1\right)\right) \\
\left.\frac{\sqrt{2}}{2} \operatorname{sgn}(y)\left(\operatorname{sgn}\left(y^{2}-x^{2}\right)-1\right)\right) \\
-\frac{\sqrt{2}}{2} \operatorname{sgn}\left(x y^{3}-x^{3} y\right)
\end{array}\right]
$$

where $\operatorname{sgn}(x)$ is the standard sign function on $\mathbb{R}$. If, however, $\mu_{S}<\mu_{R}$, then the system equations are:

$$
\left[\begin{array}{c}
\dot{x} \\
\dot{y} \\
\dot{\theta}
\end{array}\right]=\left[\begin{array}{c}
\left.\frac{\sqrt{2}}{2} \operatorname{sgn}(x)\left(\operatorname{sgn}\left(y^{2}-x^{2}\right)-1\right)\right) \\
\left.-\frac{\sqrt{2}}{2} \operatorname{sgn}(y)\left(\operatorname{sgn}\left(y^{2}-x^{2}\right)+1\right)\right) \\
\frac{\sqrt{2}}{2} \operatorname{sgn}\left(x y^{3}-x^{3} y\right)
\end{array}\right]
$$

\subsection{XY stability and $\theta$ instability}

In this section we show that the orientation $\theta$ is unstable in Equations (3) and (4). First we note that the above system is a differential inclusion of the type found in Filippov [9], i.e. we are dealing with systems of the form $\dot{x} \in F(t, x)$. For our analysis we will require the following theorem from Ref. [9]. We remind the reader that the upper and lower derivatives for a function $V(t, x) \in C^{1}$ are defined by:

$$
\dot{V}^{*}=\sup _{y \in F(t, x)}\left(V_{t}+\nabla V y\right) \stackrel{\dot{V}_{*}}{=} \inf _{y \in F(t, x)}\left(V_{t}+\nabla V y\right)
$$

Theorem 3 below from Filippov [9] is the generalization of time varying Lyapunov theory to differential inclusions.

Theorem 3 Let, in a closed domain $D\left(t_{0} \leq t<\infty,|x| \leq\right.$ $\left.\epsilon_{0}\right)$, the differential inclusion $\dot{x} \in F(t, x)$ satisfy the basic conditions of existence and $0 \in F(t, 0)$; in this domain, let there exist functions $V(t, x) \in C^{1}, V_{0}(x) \in C$ for which

$V(t, 0)=0, \quad V(t, x) \geq V_{0}(x)>0 \quad\left(0<|x|<\epsilon_{0}\right)$ Then:

I) If $\dot{V}^{*} \leq 0$ in $D$, the solution $x(t)=0$ of the inclusion $\dot{x} \in F(t, x)$ is stable.

2) If, moreover, there exist functions $V_{1}(x) \in C, W(x) \in$ $C\left(|x| \leq \epsilon_{0}\right)$ and

$$
\begin{aligned}
0<V_{0}(x) \leq V(t, x) & \leq V_{1}(x), & \dot{V}^{*} & \leq-W(x)<0, \\
(0<|x| & \left.<\epsilon_{0}\right), & V_{1}(0) & =0
\end{aligned}
$$

then the solution $x(t)=0$ is asymptotically stable.

Given that our system is not time varying, we are fortunately left with a relatively simple task. To check the $(x, y)$ translational stability of the object's motion, choose $V(x, y)=x^{2}+y^{2}$. Then

$$
\begin{aligned}
\dot{V}= & \frac{\partial V}{\partial x} \dot{x}+\frac{\partial V}{\partial y} \dot{y} \\
= & x\left(-\operatorname{sgn}(x)\left(\operatorname{sgn}\left(y^{2}-x^{2}\right)+1\right)\right)+ \\
= & y\left(\operatorname{sgn}(y)\left(\operatorname{sgn}\left(y^{2}-x^{2}\right)-1\right)\right) \\
& \quad(x \operatorname{sgn}(y)-x \operatorname{sgn}(x)) \operatorname{sgn}\left(y^{2}-x^{2}\right)- \\
\leq & 0 \quad \forall x, y
\end{aligned}
$$


Now we have to check the places where the derivative of $V$ fails to exist, i.e. where $x y=0$. First we check the case when $x=0$. Taking the upper derivative as in Equation (5), we get:

$$
\dot{V}^{*}=\sup _{\xi \in F} \nabla V \xi=y \operatorname{sgn}(y)(\xi-1) \quad \xi \in[-1,1]
$$

Therefore, $\dot{V}^{*}=0$. The case for $\mathrm{y}=0$ is computed similarly.

In the case of $\mu_{S}<\mu_{R}$, we get $\dot{V}^{*}<0$, thus implying that wheels satisfying this relationship will have a better chance of stabilizing the system. This has implications from the viewpoint of implementation of the MDMS, in that tires that favor a no rolling constraint will produce systems easier to control than tires which are designed to prevent sideways slip. Now we show that $\theta$ is unstable. We know from Equations ( 3 ) and (4) that the rotational kinematics do not depend in any way on $\theta$. $\theta$ is therefore unstable. It does turn out that the $\theta$ variable tends towards an equilibrium, but that equilibrium is dependent on the initial conditions. Simulations in Section 3.3 confirm this analysis.

\subsection{Feedback for Distributed System}

Note that the forms of Equations (3) and (4) are deceptively simple, as these equations assume that all of the actuator inputs assume a constant value. When the inputs are variable, the system equations will have the form

$$
\dot{x}=g_{\sigma_{1}} u_{1}+g_{\sigma_{2}} u_{2}+g_{\sigma_{3}} u_{3}+g_{\sigma_{4}} u_{4}
$$

All the $g_{i}$ are switched vectors as in Definition 2 which depend discontinuously on the state $(x, y)$, and therefore produce a switched system as in Definition 2. The $u_{i}$ are the control inputs at the actuator locations. In the case of $\mu_{S}>\mu_{R}$, the system equations are simply $\dot{x}=g_{i} u_{i}$ with $i$ corresponding to the index of the quadrant in which the body center of mass currently resides. Therefore the system is not even locally controllable (although it may be globally controllable) meaning that we can't hope for a simple control law. However, if $\mu_{S}<\mu_{R}$, we have governing equations of the form $\dot{x}=g_{s t a b} u_{i}+g_{\theta} u_{j}$, where $s t a b$ refers to the $(x, y)$ stabilizing property of that $g$ in whatever quadrant it is in, i.e. there exists a $V_{x y}$ satisfying the Lyapunov stability criterion from Theorem 3 in the plane. The $\theta$ refers to the fact that $\theta$ is being controlled. We can therefore design a discontinuous control law that stabilizes $\theta$, and then use the other control to stabilize the $(x, y)$ coordinates. This matches our intuition in that in the case $\mu_{S}<\mu_{R}$, we have our contact state including a control, rather than the other case where it only includes a constraint. Controls that succeed in stabilizing Equation (6) are $u_{i}=\left|u_{j}\right|+k|(x, y)|$ and $u_{j}=-k \theta$. The stability of this can be checked as in the previous section, although with somewhat more involved calculations. Simulations in Section 5 verify these calculations. Although this is by no means a generic method of obtaining a control law, we feel that this approach can be generalized to a wide class of switched systems.

\section{Generalized Theorems}

The instability results of the previous example are not an isolated phenomena, and are generalized in this section. I.e., the instability does not necessarily arise from the specific geometry of this example. In our generalization we assume that all the actuators are a finite distance apart and make point contact with the object being manipulated. The following theorem indicates that the induced instability of the programmable vector field approach can arise in more general circumstances. The proof of the theorem relies essentially upon the same sorts of calculations as found above.

Theorem 4 Given an elliptic vector velocity field $\Psi(x, y)$ : $\mathbb{R}^{2} \rightarrow \mathbb{R}^{2}$, and a discrete planar array geometry in some neighborhood of the origin, the solution to the kinematics given by the PDM is stable in $(x, y)$ and unstable in $\theta$. Moreover, if $\mu_{S}<\mu_{R}$, then such a system is stabilizable through a discontinuous feedback law.

Sketch of Proof: To see that the system is not stable in $\theta$, it is sufficient to note that $A d_{g^{-1}}$ depends only on $x$ and $y$, therefore leaving the $\theta$ dynamics with no dependence on $\theta$. This symmetry implies that the system equations are invariant with respect to initial condition in $\theta$; therefore $\theta$ is not stable. To see that the system is stabilizable, assume that the kinematics are determined at any time $t$ by two constraints at the actuator $i$ with coordinates $\left(x_{i}, y_{i}\right)$ and input speed $u_{i}$, and one constraint at the actuator $j$ with coordinates $\left(x_{j}, y_{j}\right)$ and input speed $u_{j}$. Moreover assume that $\mu_{S}<\mu_{R}$, thereby ensuring a "rolling" constraint is satisfied rather than a "slipping" constraint. Then the governing equations are:

$$
\left[\begin{array}{c}
\dot{x} \\
\dot{y} \\
\dot{\theta}
\end{array}\right]=\left[\begin{array}{c}
\frac{-\left(u_{j} y_{1}\right)+u_{i}\left(\cos (\theta) x_{1}+\sin (\theta) y_{1}\right) y_{2}}{x_{2} y_{1}-x_{1} y_{2}} \\
\frac{-\left(u_{j} x_{1}\right)+u_{i} x_{2}\left(\cos (\theta) x_{1}+\sin (\theta) y_{1}\right)}{-x_{2} y_{1}+x_{1} y_{2}} \\
\frac{-u_{j}+u_{i}\left(\cos (\theta) x_{2}+\sin (\theta) y_{2}\right)}{x_{2} y_{1}-x_{1} y_{2}}
\end{array}\right]
$$

Then, taking $V(x, y, \theta)=\left(x^{2}+y^{2}+\theta^{2}\right)$ we see that

$$
\dot{V}=\begin{aligned}
& u_{i} \frac{x_{2}\left(\cos (\theta)\left(\theta-y x_{1}\right)-y \sin (\theta) y_{1}\right)+\left(x \cos (\theta) x_{1}+\sin (\theta)\left(\theta+x y_{1}\right)\right) y_{2}}{x_{2} y_{1}-x_{1} y_{2}} \\
& -u_{j} \frac{\theta-y x_{1}+x_{1}}{x_{2} y_{1}-x_{1} y_{2}}
\end{aligned}
$$

Note that this is split into two coefficients of $u_{i}$ and $u_{j}$, which in turn implies that if we have full knowledge of the state, then we can always choose the inputs so as to make $\dot{V} \leq 0$. Moreover, if the coefficients of $u_{i}$ and $u_{j}$ are all nonzero by virtue of the configuration of actuators, we can guarantee $\dot{V}<0$, and therefore guarantee asymptotic stability. I.e., the system can be stabilized through the use of feedback. More involved calculations lead to the same result for $\dot{V}^{*}$. A possible difficulty with this result is that the stabilizing control switches discretely as the contact state switches, which suggests the need for a way of estimating the contact state. $\square$

Now the $\mu_{S}<\mu_{R}$ assumption only has an obvious physical interpretation for wheel-like contacts, and we do not yet 
have a formulation for generic contacts. The case $\mu_{R}<\mu_{S}$ may be stabilizable, but not in as straight forward a fashion. This is for largely the same reason that it may be globally controllable, but is not locally controllable, as discussed in Section 3.3.

\section{Simulations}

To illustrate these concepts, in this section we provide the results of simulations that model the system in Figure 2 based on the open loop design found in in Bohringer et al [3]. We used Mathematica to do these simulations, using its NDSolve integrator. modified to allow for hysteresis. Hysteresis is necessary in order to avoid the numerical difficulties of approaching a switching boundary (in this case $y=x, y=-x, x=0, y=0$ ).

We first simulate the open-loop control system and algorithm found in Bohringer et al [3]. That is, in this algorithm, no sensing is used to modify the actuator's preprogrammed control actions (the application of feedback to this system is described below). The simulation implements the power dissipation model applied to the true discrete actuator array system.
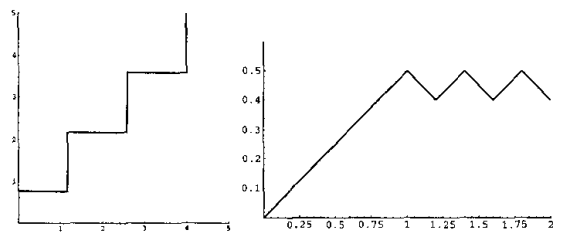

Figure 4: X,Y, and $\theta$ trajectory of non-feedback system

Figure 4 shows the simulation output when vector fields are designed to stabilize to the origin with $\theta_{0}=0$. Notice that the origin is translationally stable, as the switching system equations always satisfy the lyapunov function $V=x^{2}+y^{2}$. The simulations are done with a slight hysteresis as the dynamics approach the diagonal $y=x$. Notice that with an initial condition of $\theta_{0}=0$ (the desired $\theta$ ), $\theta$ is unstable and increases linearly until the switching begins and then hovers around a new equilibrium (not $\theta_{0}=0$ ).

Figure 5, shows the simulation results when this system is controlled using the feedback law described in the Section 3.3. Notice that the translational stability of the origin is maintained, while the rotational dynamics are stabilized to $\theta=0$ due to our feedback law.
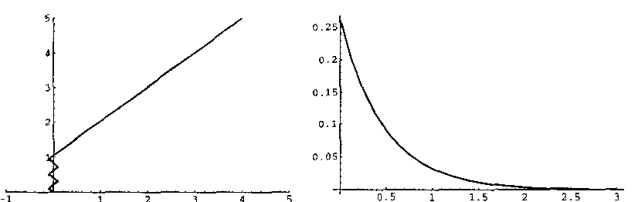

Figure 5: X,Y, and $\theta$ trajectory of feedback system

\section{Conclusions}

We found, using the power dissipation method, that the rotational dynamics of distributed manipulation systems controlled by the continuous vector field approach are unstable. This generalizes the results of Luntz et al [4] where smooth dynamics were considered. Moreover, we found a feedback law that stabilizes the system. Although our approach does require sensing, in particular it requires feedback of the angle $\theta$, the added resistance to switching of contact state is clearly worth the tradeoff. The advantage of the PDM is that it yields relatively simple model equations, whereby techniques from nonsmooth analysis can be used to understand stability issues. That our results are consistent with Luntz et al [4] suggests that much more work needs to be done to make distributed manipulators practical. Although it is clear that the programmable vector field can be expected to have nice properties far away from equilibrium, it is also clear that local dynamics must take into account contact mechanics. Moreover, the PDM gives good insight into evaluation of design philosophies, in this case leading us to believe that in the case of wheeled actuators, contact designs with $\mu_{S}<\mu_{R}$ are superior to those with $\mu_{S}>\mu_{R}$. Next we anticipate using this preliminary work to produce experimental results using a distributed array of actuators.

Acknowledgements: This work was partially supported by a grant from the National Science Foundation (grant NSF9402726) through its Engineering Research Center (ERC) program

\section{References}

[1] M.A. Erdmann and M.T. Mason. An exploration of sensorless manipulation. IEEE Journal of Robotics and Automation, 4(4), 1988.

[2] K.Y. Goldberg. Orienting polygonal parts without sensing. Algorithmica, 143(2/3/4):201-225, 1993.

[3] K.F. Bohringer, Bruce R. Donald, Lydia E. Kavraki, and Florent Lamiraux. Distributed Manipulation, chapter A distributed, universal device for planar parts feeding: unique part orientation in programmable force fields, pages 1-28. Kluwer Academic Publishers, 2000.

[4] Jonathan E. Luntz, William Messner, and Howie Choset. Distributed Manipulation, chapter Discreteness Issues in Actuator Arrays. Kluwer Academic Publishers, 2000.

[5] J.C. Alexander and J.H. Maddocks. On the kinematics of wheeled vehicles. The International Journal of Robotics Research, 8(5):15-27, October 1989.

[6] T. Murphey and J. W. Burdick. Issues in controllability and motion planning for overconstrained wheeled vehicles. In Proc Int Conf Math Theory Networks and Systems (MTNS), Perpignan, France, 2000.

[7] Howie Choset Karl F. Bohringer, editor. Distributed Manipulation. Kluwer Academic Publishers, 2000.

[8] Suresh Goyal, Andy Ruina, and Jim Papadopoulos. Planar sliding with dry friction. WEAR, 143:307-352, 1991.

[9] A.F. Filippov. Differential Equations with Discontinuous Right Hand Sides. Kluwer Academic Publishers, 1988. 